\title{
¿Es posible la reducción epistemológica? Todo sistema necesita presupuestos extra-sistémicos
}

Is epistemological reduction possible?

Every system needs extra-systemic presuppositions

José V. ORÓN

Universidad de Navarra

Grupo Mente-cerebro.

Instituto Cultura y Sociedad (ICS)

josevictororon@gmail.com
JaVIER SÁNCHEZ-CAÑIZARES

Universidad de Navarra

Grupo Mente-cerebro.

Instituto Cultura y Sociedad (ICS)

Grupo de Investigación

"Ciencia, Razón y Fe" (CRYF)

js.canizares@unav.es
Abstract: Is an epistemological reduction strictly possible? Scientific methodology claims that a boundary separating the system from the "extra-system" can be defined. However, no system defines its own limits: rather, every system needs extra-systemic presuppositions that are defined from outside the system. In this article, we show how various areas of knowledge presuppose the presence of an extra-systemic reality that provides meaning: to know any system, knowledge of the "extra-system" is also necessary.

Keywords: Epistemology, limits of knowledge, interdisciplinarity, presuppositions of science.
Resumen: ¿Resulta estrictamente posible realizar una reducción epistemológica? La metodología científica reclama que se defina un contorno que separe el sistema del "extra-sistema". Pero ningún sistema define sus límites desde sí mismo: todo sistema necesita presupuestos extra-sistémicos definidos desde fuera del sistema. En este artículo ilustramos cómo diversas áreas del conocimiento presuponen la presencia de una realidad extra-sistémica que les dé significado: el conocimiento del "extra-sistema" es necesario para conocer el sistema.

Palabras clave: Epistemología, límites del conocimiento, interdisciplinariedad, presupuestos de la ciencia. 


\section{INTRODUCCIÓN}

E 1 conocimiento científico es hoy por hoy una de las actividades definitorias del ser humano. Si bien la definición precisa de ciencia natural resulta un tema aún controvertido en el campo de la filosofía de la ciencia, puede decirse que existe un alto grado de consenso a la hora de evaluar la cientificidad de determinadas exposiciones cognoscitivas. Con independencia de su dictamen favorable o desfavorable, los referees de la mayoría de las revistas científicas saben bien qué puede considerarse un trabajo científico y qué no. Aunque de vez en cuando se produzcan fraudes o intentos de mostrar las debilidades del método de evaluación, estos no van más allá de ser excepciones que confirman la regla. El quehacer científico sigue siendo en la actualidad una de las actividades intelectuales más altas.

Uno de los rasgos determinantes del método científico es la reducción epistemológica. Se busca el menor número de hipótesis que pueda dar razón del mayor número de fenómenos. Esta reducción epistemológica ha permitido ir unificando diferentes conocimientos y ramas científicas a lo largo de la historia. Si se tiene, además, una posición realista respecto del conocimiento que nos otorga la ciencia, se obtiene la conclusión de que fenómenos aparentemente muy diversos tienen su origen en comportamientos fundamentales de la naturaleza, de los que resultan ser una instancia particular debido a otras contingencias que influyen en su realización física.

Lo que resulta menos evidente es que la reducción epistemológica inherente al método científico conlleve en último término un reduccionismo ontológico que permita explicar la emergencia de toda la realidad "de abajo a arriba". La cuestión, planteada en forma de preguntas que involucran a algunas de las ciencias contemporáneas, sería: ¿es toda la sociología reducible a antropología? ¿Toda la antropología reducible a biología? ¿Toda la biología reducible a química? ¿Toda la química reducible a física? ¿Existe una ciencia última - que podría ser la física, al tratar su rama más fundamental de las componentes básicas de la materia- a partir de la cual construir todas las demás? Si ese fuera el caso, las ciencias "construidas" 
resultarían simplificaciones convenientes para obtener leyes menos básicas, pero más cercanas a los fenómenos, que dieran cuentan de ellos en determinada escala u orden de magnitud. Las ciencias más básicas podrían dar una explicación necesaria y suficiente de lo que sucede en otros niveles de la realidad aunque, por motivos prácticos, los problemas en determinado nivel epistémico se pudieran resolver mediante leyes funcionales operando en el mismo nivel ${ }^{1}$.

Además de ilustrar la falacia de un reduccionismo o "fundamentalismo ontológico" que pretenda comprender la realidad en los términos anteriormente expuestos, el propósito de este artículo es argumentar la estricta imposibilidad de una reducción epistemológica ilimitada. La tentación del fundamentalismo ontológico sigue muy enraizada en la mentalidad de buena parte de la ciencia contemporánea, como resulta evidente, por ejemplo, de la descripción que Mangabeira Unger y Smolin hacen de los problemas que aquejan a la cosmología física actual². Mientras que la reducción epistemológica es un procedimiento necesario y altamente exitoso de la ciencia, su identificación con una perspectiva constructivista de las ciencias, asociada a una comprensión monista de la realidad, supone una inferencia equivocada desde el punto de vista lógico y una ceguera notable desde el punto de vista ontológico. No se trata ya únicamente de que toda ciencia tiene unos presupuestos irre-

1. En una contribución clásica, Oppenheim y Putnam pretendieron articular la unidad de la ciencia mediante la reducción de conceptos y leyes a aquellos más elementales. Para ambos, es creíble la unidad de la ciencia como hipótesis de trabajo; y es también plausible dada la simplicidad de las hipótesis y las pruebas directas e indirectas en su favor: cf. P. OpPEnHeIM, H. PUTNAM, Unity of Science as a Working Hypothesis, "Minnesota Studies in the Philosophy of Science" 2 (1958) 3-36. No es nuestra intención entrar a discutir en este artículo los posibles sentidos de reducción epistémica de los que se ocupa la filosofía de la ciencia.

2. "All our ideas about parts of nature will be influenced, whether knowingly or not, by our assumptions about the whole universe. Contemporary physics and cosmology have repeatedly inverted this principle": R. MANGABEIRA UngER, L. Smolin, The Singular Universe and the Reality of Time: A Proposal in Natural Philosophy (Cambridge University Press, Cambridge, 2015) xx. Así, por ejemplo, "the question of why the universe is so biofriendly is properly a cosmological question, for this fact depends on many coincidences in the choices of laws and initial conditions": Ibidem, 398. En otras palabras, no tendría sentido limitar la comprensión del fenómeno de la vida a nuestro planeta. Aunque la vida se manifieste solo de forma localizada en la actualidad, implica a la física de todo el universo. 
nunciables ${ }^{3}$, que hacen inviable su cierre epistemológico, sino que dichos presupuestos condicionan el mismo conocimiento que puede obtener cada disciplina científica. La imagen del mundo real que las ciencias nos aportan ha dejado de tener como icono la pirámide, que se va construyendo desde abajo, y resulta mejor simbolizada por un modelo de red, con nodos y conexiones más activos que otros, sin una jerarquía estática y absolutamente definida.

En los epígrafes siguientes, nos centraremos en ilustrar el modo en que el subtítulo de esta contribución "todo sistema necesita presupuestos extra-sistémicos" se cumple en las áreas de conocimiento científico actualmente más relevantes, apuntando no solo a la imposibilidad de una reconstrucción ontológica unívoca de toda la realidad, sino a las limitaciones de la reducción epistemológica de cada disciplina, que conlleva siempre una cierta interpretación global del mundo. La reducción epistemológica sigue siendo una metodología acertada para abordar los problemas de un modo científico, pero el hecho de ser conscientes de los presupuestos implícitamente adoptados ayuda a evitar la ilícita inferencia de la epistemología a la ontología que supondría abrazar el fundamentalismo ontológico.

\section{LAS MATEMÁTICAS Y EL TEOREMA DE GÖDEL}

En la historia de las matemáticas, es bien conocido el programa reduccionista presentado por David Hilbert a comienzos del siglo xx. El fin de dicho programa sería la formalización total de las matemáticas, de modo que se pudiera demostrar, desde un número finito

3. Según Jaspers, los presupuestos de la ciencia son: a) Las reglas de la lógica: es imposible pensar y conocer si se niega el principio de contradicción. Solo podemos conocer dentro de ese ámbito, por lo que sería un error querer hacer una afirmación fuera de ese ámbito; b) La ciencia se basa en el deseo de saber, pero ninguna ciencia puede demostrar la cientificidad de tal deseo. Lo requiere pero no lo demuestra; c) La elección del objeto de estudio tampoco surge de un plan científico, sino de un deseo oscuro y desconocido; d) Las ideas que generan unidad y descubren el sistema también son extra-científicas: cf. K. JASPERS, La naturaleza de la ciencia, en S. SÁnCHEZ-Migallón (ed.), La idea de la universidad (Eunsa, Pamplona, 2013) 23-55. Acerca de la conexión entre los logros de la ciencia y sus presupuestos, puede verse M. ARTIGAS, La mente del universo (Eunsa, Pamplona, 1999). 
(o una infinidad recursivamente numerable) de axiomas, mediante una serie de inferencias lógicas permitidas, la verdad o falsedad de toda proposición matemática. No tendría sentido demostrar esos axiomas, pero sí demostrar que son consistentes, es decir que no conducen a ninguna contradicción. Además, debería haber algún algoritmo para decidir si una proposición dada se deduce o no de los axiomas. Dicho programa recibió su golpe de gracia con los teoremas de incompletitud de Kurt Gödel en la década de los treinta, quien demostró que cualquier sistema de axiomas para las matemáticas desde el cual deducirlas ${ }^{4}$ sería incompleto, es decir que habría proposiciones tales que ni ella ni su negación resultan derivables de los axiomas. Como el conjunto de las proposiciones aritméticas verdaderas es obviamente completo - o es verdadera una proposición o lo es su negación-, se sigue el sorprendente resultado de que el conjunto de las proposiciones aritméticas derivadas de un sistema de axiomas nunca será igual al conjunto de proposiciones verdaderas. Gödel demostró también que cualquier sistema de axiomas de las matemáticas es incapaz de demostrar su propia consistencia y que es, además, indecidible, en el sentido antes precisado ${ }^{5}$.

El camino comenzado por Gödel ha encontrado numerosas bifurcaciones en otros problemas lógico-matemáticos íntimamente conectados. Por ejemplo, su teorema acerca de la indecidibilidad de la aritmética y de la teoría de conjuntos (es decir, de las matemáticas) ha inspirado la demostración de la indecidibilidad del problema de la parada de una máquina de Turing: no existe un algoritmo que decida si una máquina genérica se para o no. Una generalización de este último teorema es la indecidibilidad del problema de la aleatoriedad (en el sentido de no compresibilidad definido por Gregory Chaitin) de una secuencia general de números, para el que es imposible encontrar a priori un algoritmo que decida si existe un programa más sencillo que dé como output dicha secuencia de

4. Sería necesario, al menos, que se pudiera deducir a partir del sistema cierta parte irrenunciable de la aritmética llamada "aritmética elemental".

5. Ambos resultados negativos se tienen incluso en el caso de considerar solo la aritmética. 
números ${ }^{6}$. Así pues, existen números estrictamente aleatorios, pero no puede haber un algoritmo para decidir, en general, si un número dado es o no aleatorio. Las matemáticas del último siglo se hallan marcadas por una fuerte desconfianza hacia su poder lógico de construcción y determinación de verdades, lo que ha conducido a una revitalización de las controversias entre diferentes filosofías de la matemática como el platonismo, el intuicionismo y el constructivismo ${ }^{7}$ ¿Son las mismas matemáticas una mera construcción del cerebro humano evolucionado? ¿Tiene sentido hablar de una verdad universal de las matemáticas? ¿Qué criterios debe tener una demostración matemática para ser universalmente aceptada (por ejemplo, es válida la mera reducción al absurdo)? Todo sistema necesita presupuestos extra-sistémicos, y no parece que los avances que puedan darse en la fundamentación de las matemáticas lleguen a alcanzarse sin mirar más allá de sí misma en su construcción axiomática.

\section{EL PROBLEMA DE LA MEDIDA EN FÍSICA CUÁNTICA}

La física del siglo XX ha pasado por una revolución análoga a la de las matemáticas del teorema de Gödel. La física clásica newtoniana resultó sobrepasada a comienzos del pasado siglo en dos direcciones difícilmente conciliables entre sí: la teoría de la relatividad y la física cuántica. Pero mientras que la teoría de la relatividad, a pesar de sus conocidas paradojas ${ }^{8}$ resultantes de mantener constante la velocidad de la luz con independencia del estado de movimiento del sistema de referencia, sigue siendo una teoría "objetiva" (en el sentido de existir una correspondencia biunívoca entre las magnitudes físicas de los sistemas estudiados y las mediciones realizadas por los observado-

6. Cf. G. Chattin, Randomness and Mathematical Proof, "Scientific American" 232 (1975) 47; Idem, Meta Math! The Quest for Omega (Vintage Books, New York, 2005).

7. Puede verse la interesante discusión al respecto en R. Penrose, The Road to Reality. A Complete Guide to the Laws of the Universe (Jonathan Cape, London, 2004) 371-378.

8. Como que el antes y el después dependan del estado de movimiento del observador. 
res), la física cuántica rompe con dicha objetividad ${ }^{9}$. Mientras no se lleve a cabo una medida en un sistema físico, la mecánica cuántica predice que dicho sistema ha de ser descrito por una función de onda que no tiene referente directo en nuestro mundo clásico. Sin embargo, cuando se realiza una medición, la función de onda del sistema, generalmente indeterminada, pasa a ser la del estado determinado por el resultado de dicha medición ${ }^{10}$. Es decir la presencia del observador (que es extrasistémico) acaba determinando cómo se comporta el sistema. Salvo en casos muy excepcionales, solo se pueden tener probabilidades acerca del resultado de las mediciones. Lo que resulta desconcertante es que la mecánica cuántica no describe meramente nuestro desconocimiento de unas variables ocultas que pudieran estar determinando los resultados. La cuestión es, simplemente, que las magnitudes físicas observables no pueden estar ontológicamente determinadas a priori (de ahí la posibilidad de sutiles efectos no locales de interferencias cuánticas, como demuestran los experimentos del tipo $\mathrm{EPR}^{11}$ ): solo se determinan a partir de una medición.

Lo que acabamos de describir se conoce como el "problema de la medida” en física cuántica ${ }^{12}$. Mientras que esta disciplina continúa cosechando enormes éxitos en sus capacidades predictivas gracias a

9. Cf. M. Epperson, E. Zafiris, Foundations of Relational Realism. A Topological Approach to Quantum Mechanics and the Philosophy of Nature (Lexington Books, Lanham, Maryland etc., 2013).

10. Esta es la interpretación estándar (de Copenhague) de la mecánica cuántica. Un resumen de otras interpretaciones - que contienen problemas similares de fundamentación - puede verse en D. WALLACE, Philosophy of Quantum Mechanics, en D. Rickles (ed.), The Ashgate Companion to the New Philosophy of Physics (Ashgate, Aldershot; Burlington, VT, 2008) 16-98. Por simplicidad, hemos descrito el caso de una "medida ideal" que, aunque realizable, no es la única posible. La descripción de medidas no ideales queda fuera del alcance de este artículo.

11. Las siglas EPR hacen referencia al experimento mental propuesto en A. EINSTeIn, B. Podolsky, N. Rosen, Can Quantum-Mechanical Description of Physical Reality Be Considered Complete?, "Physical Review" 47 (1935) 777-780, para poner en entredicho la completitud de la mecánica cuántica. Sin embargo, los experimentos llevados a cabo apoyan la interpretación estándar: cf. A. AsPECT, P. Grangier, G. Roger, Experimental Realization of Einstein-Podolsky-Rosen-Bobm Gedankenexperiment: A New Violation of Bell's Inequalities, "Physical Review Letters" 49/2 (1982) 91-94.

12. Cf. R. PenRose, op. cit. 
un formalismo bien establecido, sus fundamentos se hallan en arenas movedizas. Desde luego, la física cuántica, en su interpretación estándar actual, echa por tierra cualquier sueño de construcción determinista de la realidad a partir de partículas e interacciones fundamentales. Pero, además, parece necesitar una intervención no trivial del observador (extra-sistémico) o posible cognoscente en la mismísima determinación de la realidad observada. Todo apunta a que el conocimiento (posible o real) de un observable del sistema -un presupuesto por principio extra-sistémico- condiciona el estado del sistema.

Si bien existen varias interpretaciones alternativas del problema de la medida, todas necesitan hoy por hoy añadir prescripciones cognoscitivas extra-sistémicas para dar un sentido global a la teoría. Aunque los procesos de decoherencia - procesos en los que el entorno del sistema tiende a suprimir los términos de interferencia de la función de onda, determinándola en el sentido de la física clásica - hacen que la física cuántica no manifieste todas sus rarezas en el mundo macroscópico, no explican sin embargo el resultado de las medidas individuales. Los expertos coinciden en señalar que dichos procesos no resuelven el problema de la medida $^{13}$; la división del mundo en un sistema más un entorno, que recibe un tratamiento científico diverso, resulta hasta cierto punto arbitraria ${ }^{14}$ y es, desde luego, extra-sistémica, pues invoca un entorno para explicar un sistema. Así pues, no tenemos un modelo físico que explique de forma completa la transición desde el mundo cuántico al mundo clásico que observamos los humanos. Y, a fecha de hoy, parece altamente improbable que dicha explicación pueda obtenerse mediante argumentos exclusivamente físicos ${ }^{15}$. La física también ha de mirar más allá de sí misma (a otras partes de la red

13. Cf. G. Bacciagaluppi, The Role of Decoherence in Quantum Mechanics, en E. N. ZalTa (ed.), The Stanford Encyclopedia of Philosophy (Winter 2012 Edition).

14. Cf. L. Smolin, Time Reborn. From the Crisis in Physics to the Future of the Universe (Houghton Mifflin, Boston-New York, 2013).

15. Cf. F. Sols, ¿Puede la ciencia ofrecer una explicación última de la realidad?, en F. Molina (ed.), Ciencia y Fe. En el camino de la búsqueda (CEU Ediciones, Madrid, 2014). 
de conceptos que entrelazan las ciencias) si quiere salir del atolladero conceptual en que se halla.

\section{El PROBLEMA DE LOS DIVERSOS NIVELES DE ACTIVACIÓN CEREBRAL}

El llamado problema mente-cerebro plantea la cuestión de cómo se relacionan la biología del cerebro y los procesos mentales de los seres humanos. Se suele dar por hecho que existe una profunda unidad entre mente y cerebro. Pero el diálogo se afronta de modo muy variado. La neurociencia es un área de investigación que requiere el concurso de diversas disciplinas. En la actualidad, no es de extrañar encontrar en ella novedades que contradicen resultados que se tenían por definitivos, porque no se han tenido en cuenta variables intrasistémicas y también extrasistémicas ${ }^{16}$. Esto plantea el problema de saber qué estamos midiendo y de cómo interpretar los resultados. No se trata de un problema de falta de datos sino de presupuestos ${ }^{17}$; falta un marco teórico que ayude a interpretar lo que se obtiene, pues no sabemos unificar todos los datos ${ }^{18}$.

Desde la misma neurociencia se reconocen limitaciones intrínsecas al sistema ${ }^{19}$. Los neurocientíficos conocen muy bien que el estudio del cerebro puede abordarse por niveles ${ }^{20}$. Un primer nivel sería el correspondiente a las meras reacciones químicas (un nivel

16. Cf. E. B. Falk, L. W. Hyde, C. Mitchell, J. Faul, R. Gonzalez, M. M. Heitzeg, J. Schulenberg, What is a representative brain? Neuroscience meets population science, "Proceedings of the National Academy of Sciences of the United States of America" 110/44 (2013) 17615-17622; R. Lent, F. A. C. AzEvedo, C. H. Andrade-Moraes, A. V. O. Pinto, How many neurons do you have? Some dogmas of quantitative neuroscience under revision, "The European Journal of Neuroscience" 35/1 (2012) 1-9.

17. Cf. S. Firestein, Ignorance: How it Drives Science (Oxford University Press, New York, 2012); H. MARKRAM, Seven challenges for neuroscience, "Functional Neurology" 28/3 (2013) 145-151.

18. Cf. Ibidem; O. SPORNS, Contributions and challenges for network models in cognitive neuroscience, "Nature Neuroscience" 17/5 (2014) 652-660.

19. Cf. S. Firestein, op. cit.; H. Markram, op. cit.; O. SpOrns, Contributions and challenges cit.

20. Cf. C. Blanco, Historia de la neurociencia. El conocimiento del cerebro y la mente desde una perspectiva interdisciplinar (Biblioteca Nueva, Madrid, 2014) 135. 
molecular). Un segundo nivel viene determinado por la unidad de la neurona. El tercer nivel se asocia a las diversas redes que se descubren en el funcionamiento del cerebro. El cuarto, con las relaciones inter-redes que se asocian a diversas funciones. Y habría también un quinto y sexto niveles - ya plenamente psicológicos- que estudian procesos como la toma de decisiones y el comportamiento global y la personalidad, respectivamente. Ahora bien, siempre que se estudia un nivel se hace desde la conciencia de que se va a acabar incidiendo en otros niveles. Cada nivel engloba especialistas que pueden dedicar toda su vida a subniveles internos sin agotarlos. Se evidencia la irreductibilidad de unos niveles a otros, de tal forma que no sabemos cómo conectarlos: nos faltan “escaleras"21. Pero además, se podrían tener en cuenta todos los niveles que afectan al ser humano; desde el nivel genético al social ${ }^{22}$. Por ello, se apuesta cada vez más por hacer una neurociencia integradora ${ }^{23}$ donde incluso puedan acogerse niveles espirituales ${ }^{24}$.

El cerebro es un sistema complejo que se caracteriza por: (a) unos patrones con altos niveles de conectividad; b) una organización multiescalar a varios niveles; c) comportamientos dinámicos no-lineales; d) resiliencia ante cambios externos; e) una capacidad de autoorganización con fenómenos de memoria ${ }^{25}$. Todo ello ilustra una dinámica de funcionamiento cerebral que aboga por la presencia de una realidad extra-sistémica. Si en el cerebro la estructura determinara unidireccionalmente la función, no podría entenderse cómo algo extra-sistémico puede incidir en sus funciones. En cambio, descubrimos que estructura y función se influyen bidireccionalmente ${ }^{26}$,

21. Cf. S. Firestein, op. cit.; H. Markram, op. cit.

22. Cf. E. B. FALK et al., op. cit.

23. Cf. E. GoRDON, Integrative neuroscience, "Neuropsychopharmacology" Suppl 1 (2003) S2-S8; S. H. KosLOW, Discovery and integrative neuroscience, "Clinical EEG and Neuroscience" 36/2 (2005) 55-63.

24. Cf. M. J. FARAH, Neuroethics: the ethical, legal, and societal impact of neuroscience, "Annual Review of Psychology" 63 (2012) 571-591.

25. Cf. O. SpORns, The human connectome: a complex network, "Annals of the New York Academy of Sciences" 1224 (2011) 109-125.

26. Cf. D. S. Bassett, M. S. GaZZANIGA, Understanding complexity in the buman brain, "Trends in Cognitive Sciences" 15/5 (2011) 200-209; O. SPORNS, Contributions and challenges cit. 
habiendo modificaciones en el mismo comportamiento ${ }^{27}$. La misma dinámica cerebral permite de una forma suave que la interacción extra-sistémica pueda alcanzarle naturalmente.

\section{UNA PSICOLOGÍA ABIERTA QUE RECLAMA SER TRANSCENDIDA}

Desde el campo de la psicología también se pueden encontrar líneas de investigación que reclaman una presencia extra-sistémica para poder dotar de coherencia a todo el sistema. Nos referimos a los estudios sobre el comportamiento ético de la persona y su desarrollo madurativo en dos autores cuyas formulaciones son ampliamente aceptadas y utilizadas como referentes: Lawrence Kohlberg ${ }^{28}$ y Erik Erikson ${ }^{29}$. Kohlberg ${ }^{30}$ realizó su aportación más importante en el desarrollo moral de la persona y Erikson ${ }^{31}$ en su desarrollo global, haciendo hincapié en cómo el ser humano se enfrenta a diversos retos de crecimiento en cada época de su vida.

Kohlberg ${ }^{32}$ plantea que el desarrollo moral del ser humano empieza desde su más tierna infancia. La moral trata de responder a la pregunta sobre qué acciones son buenas, y Kohlberg señala cómo esa pregunta es respondida de diversas formas en diversos estadios del crecimiento. Considera en concreto un desarrollo preconvencio-

27. Cf. O. SpORns, Contributions and challenges cit.

28. Cf. J. Greene, J. HaIDT, How (and where) does moral judgment work?, "Trends in Cognitive Sciences" 6/12 (2002) 517-523; H. K. MA, The moral development of the child: an integrated model, "Front Public Health" 18/1 (2013) 57; D. NARváEZ, Human Flourishing and Moral Development: Cognitive and Neurobiological Perspectives of Virtue Development, en L. NuCCI, E. NARVÁEZ (eds.), Handbook of Moral and Character Education (Erlbaum, Mahwah, NJ, 2008) 310-327; J. Rest, C. PowER, M. BRABECK, Lawrance Koblberg (1927-1987), "American Psychologist” 43/5 (1988) 399-400.

29. Cf. E. Douvan, Erik Erikson: critical times, critical theory, "Child Psychiatry and Human Development” 28/1 (1997) 15-21; L. J. FrIEDMAN, Identity's Architect: A Biography of Erik H. Erikson (Scribner, New York, 1999); J. MARCIA, R. JosseLsON, Eriksonian Personality Research and Its Implications for Psychotherapy, "Journal of Personality" 81/6 (2013) 617-629.

30. Cf. L. Kohlberg, The Claim to Moral Adequacy of a Highest Stage of Moral Judgment, "Journal of Philosophy" 70/18 (1973) 630-646.

31. Cf. E. H. Erikson, El ciclo vital completo (Paidós, Barcelona, 1997).

32. Cf. L. Kohlberg, op. cit. 
nal, un desarrollo convencional y un desarrollo postconvencional, cada uno de los cuales se divide a su vez en dos subestadios. En el estadio preconvencional, el criterio para responder a la pregunta moral es el propio interés: mi bien es el bien. En el convencional, el criterio es el bien del grupo (amigos o sociedad); el bien que permite que yo sea parte de ellos es mi bien. Hasta ese nivel llegan también los chimpancés. En el estadio postconvencional, el criterio se conceptualiza y el bien pasa a ser lo bueno en sí, llegando a alcanzar referencias como los derechos humanos y la valoración de ciertos principios como salvaguarda de la humanidad. El propio Kohlberg ${ }^{33}$ añade finalmente un séptimo estadio que califica de orden metafísico, necesario para la psicología, donde desarrolla una teoría de la ley natural, al descubrir que el ser humano no puede ser la instancia que se dé a sí misma los principios del actuar: pueden ser descubiertos por la razón, pero no son creados por ella. Y para desarrollar su teoría acude a Marco Aurelio y a Spinoza. Saberse formando parte de algo más grande que uno mismo implica que el que actúa contra otro actúa contra sí mismo, al formar parte ambos de la única realidad. Kohlberg descubre la necesidad de un amor eterno como lo único que llega a saciar al ser humano, de ahí su llamada a la eternidad. También rechaza la falacia naturalista de pensar que lo bueno existe en sí. La moral que declara únicamente lo bueno en sí, no será nunca una respuesta al porqué de la moral. Ser moral solo puede responderse desde fuera de la moral, pues requiere responder a la pregunta acerca de qué significa ser un ser humano. Si el ser humano no tiene significatividad, no hay base para la moral. Pero esa significatividad no se la otorga él mismo.

Erikson a su vez plantea un crecimiento del ser humano que se juega en retos y dilemas ${ }^{34}$. Cada edad tiene su punto de crecimiento y según como se resuelva ese planteamiento se crecerá de una forma u otra. Si no se resuelve bien, se producirá un déficit personal que se arrastrará toda la vida y no será superado hasta que no sea aten-

33. Cf. L. Kohlberg, R. A. RynCARZ, Beyond fustice Reasoning: Moral Development and Consideration of Seventh Stage, en E. Langer, C. N. AleXander (eds.), Higher stage of human Development (Oxford University Press, New York, 1990) 191-207.

34. Cf. E. H. ERIKSON, op. cit. 
dido adecuadamente, pues el tiempo solo no cura. Plantea la sanidad en términos de integración holística de toda la persona, por lo que ninguna época es desdeñable en ese sentido. Pero apunta que esa integración no se queda en sí, describiendo como un último estadio la "gerotrascendencia". Hasta el inicio de la adolescencia, Erikson asocia los retos a edades, mas a partir de entonces ya no se puede hacer una asociación rígida a las edades pues, aunque se observa que los mayores estadios se suelen dar a mayor edad, estamos ya ante una cuestión muy variable interpersonalmente. Por ello, ya no habría que tomarlos como retos propios de la edad sino como camino de maduración humana. En ese sentido la gerotrascendencia no sería un problema ligado a la ancianidad, sino a la plenitud de la maduración. Y así lo entiende al empezar su libro precisamente por esta época de "identidad existencial" que integra pasado, presente y futuro. En ella, las personas vuelven a comprender de una forma nueva todo lo vivido asimilando muchos procesos distónicos. Pero si la persona resuelve positivamente las distonías propias de la edad $^{35}$ descubrirá "la intensidad de ser y [podrá] esperar una mayor gracia e iluminación, [y con ello tendrá] una razón para vivir" " "La gerotrascendencia es un cambio en la metaperspectiva de una visión materialista y racional a una más cósmica y trascendente, acompañada, por lo general, de un incremento de satisfacción vital". "El gerotrascendente experimenta un sentimiento nuevo de comunión cósmica con el espíritu del universo, una redefinición del tiempo, la vida y la muerte, así como una redefinición del yo" ${ }^{37}$. Así pues, tanto en Kohlberg como en Erikson, el desarrollo psicológico presupone una realidad extra-sistémica, de modo que pueda tener sentido todo el crecimiento moral y el desarrollo madurativo personal.

35. Las distonías de la vejez del noveno estadio tienen el denominador común de la debilidad y la pobreza personal, que se hacen patentes y van a ir creciendo. En este momento, todos los dilemas que se han planteado a lo largo del crecimiento se vuelven a plantear, pero sabiendo que ya no se van a resolver como antes. Hace falta una forma nueva de reinterpretar la propia vida: cf. Ibidem, 109-117.

36. Ibidem, 117.

37. Ibidem, 127. 


\section{LA AMPLIA MIRADA DE LA TEORÍA DEL CONOCIMIENTO}

Mientras que el trabajo de Piaget $^{38}$ supuso un gran avance en el conocimiento de la maduración del pensamiento del niño y del adolescente, podemos encontrar, por otro lado, un planteamiento muy completo y sistémico de la teoría del conocimiento en Leonardo Polo $^{39}$. Polo afirma que también hay un crecimiento madurativo en el pensamiento adulto, de forma análoga a como Piaget muestra el crecimiento en la forma de pensar de los niños ${ }^{40}$. No es solo que se piensen más cosas o más eficientemente, sino que también se progresa de forma distinta en el pensamiento, mediante el conocimiento habitual. Polo entiende el conocimiento habitual adquirido como el conocimiento de la actividad intelectual ejercida, y expone que el crecimiento en el conocimiento lleva a un progresivo abandono del límite mental ${ }^{41}$, que invita a un salto a la transcendencia.

En muchas teorías del conocimiento se da un momento en el que se alcanza la formulación de los conceptos abstractos; momento en que se conoce la realidad en cuanto tal, aunque no seamos capaces de explicitarla totalmente. A partir de ese momento Polo sostiene que el pensamiento puede proseguir, pero por vías diferentes, que muchos filósofos confunden y él diferencia para acabar unificando. Una vía de prosecución es la de la "generalización" que conduce, mediante la negación de la diferencia entre abstractos, a las ideas generales. Esta vía es plenamente objetual. Es todo un ejercicio mental que puede proseguir hasta el infinito, pues puede ir realizando más y más generalizaciones y obteniendo más y más ideas generales para jerarquizarlas según el grado de generalización. Esta vía, insistimos, no abandona nunca la presencia mental u objetividad. La segunda vía de crecimiento del conocimiento es una vía que denomina ra-

38. Cf. B. InHELDER, J. Piaget, The growth of logical thinking from childhood to adolescence (Basic Books, New York, 1958).

39. Cf. L. Polo, Curso de teoría del conocimiento. Tomos I, II, III y IV (Eunsa, Pamplona, 2006).

40. Se trata de perspectivas diversas, pues Piaget realiza su investigación desde una perspectiva psicológica, mientras que Polo lo hace desde una perspectiva filosófica.

41. El límite mental es el término técnico Polo emplea para referirse a la presencia mental que permite la objetividad. 
cional, en la que ya no se avanza por el contraste entre ideas sino por la confrontación con la realidad. Dicha confrontación permite descubrir las causas reales extra-mentales objetivamente conocidas y avanzar hasta su fundamentación. En la medida en que se conoce más de esa realidad, se ve que la misma limitación mental descubre la presencia de la realidad: el mismo pensamiento descubre la antecedencia de lo real. Se trata de un camino de prosecución que permite abandonar el límite mental y alcanzar los primeros principios y la fundamentación de la realidad; un camino de crecimiento que descansa en un "conocimiento habitual", diverso de las operaciones intelectuales. Se acaba abandonando el límite del ejercicio mental para alcanzar una transcendencia, previamente implícita, que el conocimiento habitual advierte. Tal trascendencia requerirá la consideración de una antropología transcendente del ser humano digna de su forma de conocer.

¿En qué medida resultan válidas dichas afirmaciones para la epistemología científica? La filosofía de la ciencia es bien consciente de que toda ciencia acude a una realidad extra-sistémica que la fundamenta: unos presupuestos. Polo descubre que para que una ciencia sea estricta debe enunciar con claridad los principios y conceptos en los que se apoya, formular hipótesis de forma coherente con esos principios y emplear después el conocido método científico ${ }^{42}$. Ahora bien, podemos encontrar rastros del conocimiento habitual que Polo señala en formas de conocer como el asombro, la contemplación de la naturaleza o el conocimiento mediante analogías ${ }^{43}$ e intuiciones. Hay un conocimiento en la ciencia que no es meramente el resultado del método científico ${ }^{44}$. Las ciencias también conocen

42. Cf. Ibidem I, 92 (la paginación es respecto al volumen I por separado).

43. El uso de la analogía en ciencia es una fuente exitosa de formación de hipótesis pues "la naturaleza emplea los mismos medios para iguales fines": S. RAMON Y CAJAL, Reglas y consejos sobre la investigación científica (Espasa, Madrid, 1898) 137138.

44. Ramón y Cajal, por ejemplo, abunda en esta cuestión al hablar de la intuición, del asombro, de la contemplación, de la revelación... que no se dan sin el esfuerzo racional, metódico y perseverante: "a fuerza de tiempo y de atención, el intelecto llega a percibir un rayo de luz en las tinieblas del más abstruso problema": Ibidem, 57. Otras citas similares se hallan en las páginas 56, 88, 91, 116, 121, 131, 137. 
de forma "extra-metódica". Esta pluralidad de modos de conocer ha sido enriquecedora para la misma ciencia, permitiendo la apertura de nuevos campos ${ }^{45}$. Así pues, desde una teoría del conocimiento proveniente de la filosofía y desde el mismo proceder de la ciencia se reclama la presencia extra-sistémica.

\section{ConClusiones}

El recorrido por las diversas disciplinas del conocimiento que acabamos de hacer muestra la necesidad de los presupuestos extra-sistémicos para la propia fundamentación del conocimiento. No se está reclamando simplemente un necesario intercambio interdisciplinar. Tampoco se pretende decir que haya que explicitar continuamente los presupuestos para resolver los problemas científicos concretos de una disciplina. Lo que hemos pretendido ilustrar es que una interpretación realista y profunda de lo conocido no debe prescindir de la realidad extra-sistémica en la que, en último término, se basa como condición de posibilidad. Sin la consideración extra-sistémica, el sistema de estudio carece de sentido, pues los presupuestos son reclamados por el mismo sistema. Así pues, toda pretensión de fundamentalismo ontológico en la construcción de la realidad "desde abajo" carece de sustento.

Toda reducción epistemológica tiene sus límites, que apuntan, en definitiva, a la imposibilidad de comprender plenamente la realidad de manera reductiva. Como argumentan Mangabeira Unger y Smolin, una teoría científica exacta solo podría obtenerse, en última instancia, extendiendo la descripción del sistema para que incluya todos los grados de libertad con los que el sistema interactúa, lo que

45. Por ejemplo, cuando desde nuevas ramas de una ciencia se ha acudido a la analogía con fenómenos de ramas más desarrolladas para la formulación de hipótesis. De forma similar a la analogía podríamos hablar de la intuición: muchos avances de la ciencia se han dado gracias a intuiciones de científicos en su época de juventud, que sirvieron para formular las bases de sus teorías. Dichas intuiciones necesitarán ciertamente luego de la constancia y el método para llegar a ser un aporte a la sociedad, como muestran los ejemplos de Newton, Davy, Faraday, Hertz o Mayer entre otros: cf. Ibidem, 35. 
supone incluir a todo el universo ${ }^{46}$. Pero, cabría preguntarse, ¿cuáles son y cómo se determinan esos grados de libertad? ¿Podemos pretender una comprensión científica completa del universo siendo una parte del mismo? Hacer ciencia supone una cierta reconstrucción y una cierta elección; y parte esencial de la elección es el interés del ser humano acerca de una dimensión específica de la realidad y sobre cómo se desea conocerla. La reducción epistemológica, estrictamente hablando, no es posible: nos encontramos siempre ante opciones ineludibles de comprensión global, pues todo intento de conocimiento parcial remite, en el fondo, a una interpretación global.

A lo largo de este artículo, hemos pretendido ilustrar una serie de aspectos que reclaman la consideración extra-sistémica desde diversas áreas del conocimiento: matemáticas, física, neurociencia, psicología y filosofía. La filosofía de la ciencia debería seguir avanzando en el estudio de las relaciones entre el sistema y sus presupuestos en los más diversos contextos científicos. Aquí nos limitamos a afirmar la necesidad de que tal conditio sine qua non epistemológica sea considerada, especialmente a la hora de extraer consecuencias ontológicas. Ningún sistema se entiende completamente desde sí mismo: el conocimiento del "extra-sistema" es necesario para conocer el sistema. Este recorrido interdisciplinar, necesariamente rápido, evidencia que no estamos ante un déficit de conocimiento o un problema sectorial, sino ante una propiedad intrínseca del mismo conocimiento humano. Esta conclusión puede entenderse también como una crítica al programa filosófico del materialismo, ya que la misma ciencia se reconoce fundamentalmente incapaz de explicar de modo autónomo toda la realidad que le concierne ${ }^{47}$.

46. Cf. R. Mangabeira Unger, L. Smolin, op. cit., 375.

47. Los autores desean agradecer las sugerencias recibidas de F. Sols, I. Sols, J. Bernácer y J. I. Murillo. Este trabajo ha sido posible con el apoyo económico de "Obra Social La Caixa”. 
